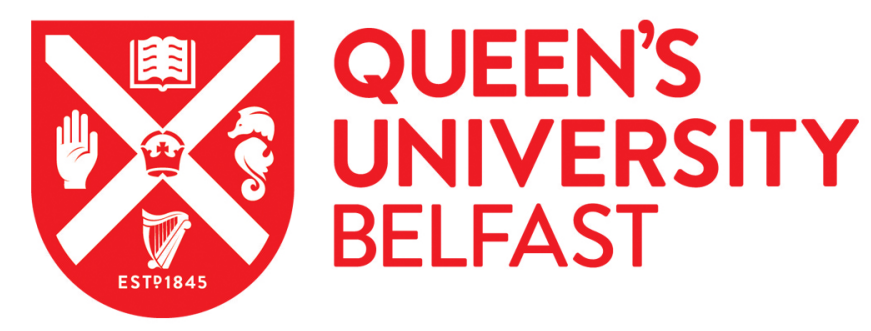

\title{
User-centred design of an active computer gaming system for strength and balance exercises in older adults
}

Howes, S., Charles, D., Pedlow, K., Wilson, I., Holmes, D., Chaponneau, G., \& McDonough, S. M. (2019). Usercentred design of an active computer gaming system for strength and balance exercises in older adults. Journal of Enabling Technologies, 13(2), 101-111. https://doi.org/10.1108/JET-12-2018-0057

Published in:

Journal of Enabling Technologies

Document Version:

Peer reviewed version

Queen's University Belfast - Research Portal:

Link to publication record in Queen's University Belfast Research Portal

Publisher rights

Copyright 2019 The Author. This work is made available online in accordance with the publisher's policies. Please refer to any applicable terms of use of the publisher.

\section{General rights}

Copyright for the publications made accessible via the Queen's University Belfast Research Portal is retained by the author(s) and / or other copyright owners and it is a condition of accessing these publications that users recognise and abide by the legal requirements associated with these rights.

Take down policy

The Research Portal is Queen's institutional repository that provides access to Queen's research output. Every effort has been made to ensure that content in the Research Portal does not infringe any person's rights, or applicable UK laws. If you discover content in the Research Portal that you believe breaches copyright or violates any law, please contact openaccess@qub.ac.uk. 


\title{
User-centred design of an active computer gaming system for strength and balance exercises for older adults
}

\begin{abstract}
Purpose: Active computer gaming (ACG) is a way for older people to participate in strength and balance exercise. Involving older adults in the development of a bespoke ACG system may optimise its usability and acceptability. The aim of this study was to employ user-centred design to develop an ACG system to deliver strength and balance exercises, and to explore its safety, usability and acceptability in older adults.
\end{abstract}

Design/methodology/approach: This paper describes user involvement from an early stage, and its influence on the development of the system to deliver strength and balance exercises, suitable for display on a flat screen or using an Oculus Rift virtual reality headset. It describes user testing of this ACG system in older adults.

Findings: Service users were involved at two points in the development process. Their feedback was used to modify the ACG system prior to user testing of a prototype of the ACG system by $n=9$ older adults. Results indicated the safety, usability and acceptability of the system, with a strong preference for the screen display.

Research limitations/implications: The sample size for user testing was small; however, it is considered to have provided sufficient information to inform the further development of the system.

Practical implications: Findings from user testing were used to modify the ACG system. This paper identified that future research could explore the influence of repeated use on the usability and acceptability of ACG in older adults.

Originality/value: There is limited information on the usability and acceptability virtual reality headsets in this population.

\section{INTRODUCTION}

Within the ageing population, reduced participation in daily activities and a reduction in physical activity lead to deconditioning, impaired function and reduced independence. Physical and cognitive decline in older age is additionally associated with an increased risk of falls. It is estimated that approximately a third of adults aged 65 and over will fall each year, making falls the greatest cause for hospitalisation in older adults (Masud \& Morris 2001; Finucane et al. 2014). Guidelines recommend strength and balance exercises, completed in standing position, to reduce the risk of falling in this population (Campbell and Robertson 2007; National Institute for Health and Care Excellence 2013). It is estimated that up to $42 \%$ of falls can be prevented by well-designed exercise programmes, such as the Otago Exercise Programme (Sherrington et al. 2011) which is an individualised, home-based programme with strength and balance exercises that can be progressed in difficulty (Gardner et al. 2001).

Active computer gaming (ACG) is becoming recognised as a potentially safe and enjoyable way for older people to participate in exercise and activities that may otherwise be difficult (Bleakley et al. 2015). There is also potential for older adults to practice these exercises unsupervised at home. Findings from a systematic review and meta-analysis of 35 randomised controlled trials (Howes et al. 2017) indicated that ACG was a safe mode of exercise for older adults with positive effects on physical and cognitive health outcomes including balance. However, it was notable the lack of studies of people at risk of falling, with most ACG interventions conducted with healthy older adults $(n=23 / 35$, $66 \%$ ) in a clinical environment with supervision. Additionally, most of the included studies investigated commercially available gaming systems $(n=23 / 35,66 \%)$, such as the Nintendo Wii and Xbox Kinect. However, as these games are developed for healthy adults, they do not always match the ability of older adults or meet their therapeutic needs.

Technologies delivering fully-immersive virtual experiences, such as virtual reality (VR) headsets, are becoming more accessible and affordable. These could provide older adults with a more immersive and realistic virtual environment (Lu and Mattiasson 2013; Howard 2017), potentially 
influencing their enjoyment and allowing them to experience activities that may not otherwise be possible. The use of VR headsets in healthcare has included the management of a number of types of conditions: physical, for example, upper limb rehabilitation post-stroke (Holmes et al. 2016); cognitive, for example, with Alzheimer's disease (García-Betances et al. 2015) and autistic spectrum disorder (Newbutt et al. 2017); psychological, for example, anxiety, phobias and eating disorders (Riva et al. 2016). Most of these conditions are suited to treatment whilst in sitting position. Some studies have recently explored the use of fully-immersive VR performed in standing position, walking on the spot and treadmill walking in healthy participants (Nilsson et al. 2016; Yoo and Kay 2016). In a study investigating treadmill walking in a fully immersive VR environment, healthy older adults $(n=11$, mean age $66 \pm 3$ years) were capable of using immersive VR with minimal adverse effects, although as expected were more dynamically unstable than their younger counterparts (Kim et al. 2017). Research to date reports limited information on the usability and acceptability of immersive VR in this population.

Bespoke ACG systems can be specifically designed to deliver tailored rehabilitation exercises, such as the Otago Exercise Programme, to meet the ability and needs of older adults. Input from end users early in the design and development phase of an ACG system is one way to optimise its usability and acceptability. Older adults face a variety of challenges when engaging with ACG technologies. One study described challenges related to physical changes, cognitive changes and self-efficacy; stating that many of these could be avoided by involving older adults in the design and testing of such systems (McLaughlin et al. 2012). User-centred design (UCD) is used in software development to optimise usability of a system as rapidly as possible; it includes task analysis, usability testing, observations and feedback from users (Fisk et al. 2009). UCD has been used in the development of VR interventions for a number of rehabilitative aims, including stroke rehabilitation (Standen et al. 2011) and for students with intellectual disability (Lanyi et al. 2012). However, there is limited information published in the area of UCD for the development of rehabilitative technologies delivering falls prevention exercises for older adults.

Previous work has discussed UCD methods in the development of ACG systems for older adults. One previous research team conducted workshops with older people to discuss requirements, brainstorm and sketch ideas, followed by a games session giving older adults the opportunity to play an initial prototype (Uzor et al. 2012). Proffitt and Lange (2013) described an iterative process involving an interdisciplinary team and stakeholders in the design and development of a system for falls prevention. This included focus groups to explore barriers and facilitators to engagement, iterative user testing of prototypes and semi structured interviews to explore user experience. More recently, a protocol for UCD for ACG for older adults has been suggested (Brox et al. 2017). This protocol includes: gathering requirements from the literature, background information on the population, discussions about their requirements and observations of their use with commercial games; an iterative design and implementation process influenced by user feedback during observations, structured and semi-structured interviews and discussions; and an evaluation phase, involving piloting of the final prototype with new participants (Brox et al. 2017).

The aim of this study was to employ user-centred design methods to develop an ACG system to deliver strength and balance exercises, for display using two viewing mediums. The aim of the user testing was to explore its safety, usability and acceptability, and older adults' perceptions of the two viewing mediums, during user testing with older adults.

\section{METHODS}

The research outline for the development of the first prototype of this system is summarised in Figure 1; the first prototype was developed for viewing on flat screen (study condition A) and using an Oculus Rift VR headset (study condition B). This study was approved by the Office for Research Ethics Committees Northern Ireland and two day care centres based in urban areas of the city agreed to participate.

\subsection{Development of the ACG system}

The design and development of the system was an iterative process involving an interdisciplinary team of clinicians and developers. Both managers of day centres for older people and service users were actively involved in the study from an early stage, influencing the study design and the development of the system. During the information gathering stage, meetings were conducted with managers of two Age $\mathrm{NI}$ day centres for older adults (Figure 1, PPI A). The information gathered provided an understanding of the service provided by the centres, including the number of days it operated, the daily routine and the activities provided. Day centre managers also shared information 
about their service users, including the number of service users in total and daily, their level of function and needs, and their activity preferences. Older adults were referred to the day centres due to physical or social needs, and attended between one and five days per week, depending on their needs. The users of both centres enjoyed playing a variety of games, such as boccia and quoits, and arm chair exercises; one of the centres had purchased a Nintendo Wii, however, this was never used by any of the users as the staff were not familiar with its use.

Service users were involved at two points in the design and development process. Service users $(n=25)$ were consulted during the early stages of development (Figure 1, PPI B). During three scoping meetings, they were shown images and given information about the technology and its potential for a falls prevention intervention, and given the opportunity to provide feedback. Handwritten notes were made on their questions, views, concerns and recommendations. Feedback was generally positive, with the service users showing interest in the novel technology. They described seeing family members play such games, and one stated they had seen VR headsets used in a TV programme. Many said they would be willing to participate, but that they would not be willing and/or able to travel to the university to take part. The outcomes of the meetings led to the decision to conduct the testing within the day centres rather than in the University Research Centre.

Later in the development process, when an initial prototype was available, two meetings were conducted with service users $(n=18)$ to discuss the game design and choice of exercises (Figure 1, PPI C). During small workshop style meetings, older adults were given information about the ACG system and asked to provide feedback in order to refine the system. Many of the service users spoke about arthritis and osteoporosis as barriers to exercise, sometimes referring to having a good side and a bad side, and that their ability to complete the exercises would differ left/right accordingly. The service users, for the most part, thought that the other exercises seemed easier, and some stated that they completed similar exercises at home, prescribed by physiotherapists for hip or knee pain or following orthopaedic surgery. Many expressed that they would prefer completing the exercises with a chair for support, to improve their confidence in performing the exercises. Most of the service users seemed very interested and keen to try the games, while some of the service users asked if they could try the game now, or said they would stand up to see if they could do the individual exercises. This provided a good indication that they may be willing to take part in the user testing when it commenced. During these meetings, the service users were also consulted about the presentation of the game. Images of screen grabs from the game were shown to the users in small groups of 2-3, and they were given the opportunity to provide feedback on their perceptions of the game design, colours, clarity and ease of reading of the text. This included use of colour and ease of reading. Day centre service users all stated that they could read the instructions and print within the games easily. They felt that all the colours were easy to see and read. Several service users reported that they thought the font size used on the score sheet at the end was too small and that too much information was presented. Comments and suggestions were fed back to the research team and implemented as appropriate.

\subsection{Description of the ACG system}

The VR content, based on the exercises in the Otago Exercise Programme, was developed using Unity 3D software (Unity Technologies SF Inc., San Fransisco, CA, USA). The software ran on an Alienware PC (Alienware Corps., Miami, FL, USA.) connected to a Microsoft Kinect Camera (Microsoft Corps. Redmond, WA, USA.) mounted on a tripod positioned at $85 \mathrm{~cm}$ above desk height, to track user movements. The VR content was developed for display using the two viewing mediums, a 21 inch monitor (screen condition A) and the Oculus Rift head-mounted display (Oculus VR., Irvine, CA. USA.; VR condition B). The purpose of this was to explore older adults' perceptions of the two viewing mediums.

The exercises in the Otago Exercise Programme were shared with the development team and the technical difficulty of developing a game that included these exercises was discussed. The ACG system included four mini-games to deliver exercise tasks based on four exercises included in the Otago Exercise Programme (Province et al. 1995): Knee Bends, the user bends their knees to duck under passing logs ( $n=10)$; Leg Abduction, the user raises their leg to strike balls positioned to the side ( $n=10$ each side); Sideways Walking, the user steps to the side to avoid oncoming walls $(n=10)$; One Leg Stand, the user stands on one leg to avoid rising water ( $3 \times 10$ seconds each leg) (Figure 2). In each mini-game, the Kinect camera tracked the participant displaying their bodily movements on the screen as a white figure. At the beginning of each game, the participant was instructed to stand on the yellow " $X$ " to allow for calibration; the Kinect used this position as a reference point to track users' movements and success in each task. Additionally, prior to beginning the Knee Bends game, the 
height of the logs was calibrated to the height of the participant. The ACG system was developed for display on two viewing mediums - on a flat screen and with an Oculus Rift VR headset.

As per feedback provided during the meetings with older adults, the system was developed to be able to detect the movements of the user with a zimmer frame placed in front to enable participants to use their own walking aid for hand support where required. The purpose of this was threefold: to improve participants' confidence, as falls efficacy scores indicated that most participants had high concern about falling; to enable them to play despite physical limitations, and it was expected that some may have low exercise tolerance due to de-conditioning; and, to ensure safety, so that participants could reach for hand support should they lose their balance.

\subsection{User testing methods}

Participants were recruited through the two Age NI day centres. The study was carried out over one (screen condition only) or two visits (screen and VR conditions); and conducted in the day centre that the participant attended. Individuals were eligible for inclusion if they were aged over 65 years, had stable health. and were independently mobile with or without a walking aid. The eligibility criteria were chosen to be as inclusive as possible; however, as the exercises required to play the game were performed in standing position, it was necessary to exclude older adults that were not independently mobile such that they were bed or wheel chair bound. Older adults with a current acute or uncontrolled medical condition or significant cognitive impairment were excluded; these were screened using the Physical Activity Readiness Questionnaire (Thomas et al. 1992) and the Mini Mental State Examination (Folstein et al. 1975), respectively.

\subsubsection{Procedure}

Two researchers were present throughout testing. One researcher demonstrated the use of the system and mini-games, while the other highlighted key features of use and gave the participant the opportunity to ask any questions. Participants were advised to ask for any verbal or physical assistance that they may require at any stage, and to make comment with regards to their ability to complete each task and difficulty experienced doing so. Sessions were video recorded to supplement hand-written notes made during participants' use of the system.

The protocol had outlined a plan for randomisation of the order that study conditions were presented to the users. However, following piloting the VR condition in healthy adults, it was decided to introduce the system to older adults using the screen display for familiarisation prior to using the VR headset display.

\subsubsection{Outcomes of interest}

Safety: A safety checklist pro-forma was completed for each participant, to document both safety components and practical aspects of using the equipment during participants' use of the system.

Usability: Details of additional verbal and physical assistance required, as well as any participant comments were recorded on the safety pro-forma. The System Usability Scale (SUS), a reliable and valid measure of perceived usability (Brooke 1996), was completed by participants after each study condition. This scale comprises 10 items which are rated on a 5-point Likert scale ranging from 1 "strongly disagree" to 5 "strongly agree" to subjectively evaluate their perceptions of the ease of use and usability of the system. Scores above 70 indicate acceptable usability, while scores below 50 indicate unacceptably low usability (Bangor et al. 2008). Comparison of usability scores for each study condition were used to compare older adults' perceptions of using the two viewing mediums.

Acceptability: Acceptability was measured using the Attitudes to Falls-Related Interventions Scale (AFRIS). The scale includes 6 items which are rated on a 7-point Likert scale ranging from1 "strongly disagree" to 7 "strongly agree". The AFRIS items consider the following components of acceptability: attitudes; subjective norm; perceived behavioural control; identity; and, intention (Yardley \& DonovanHall 2006 \& 2007). Responses to the individual items of the AFRIS and any comments made by participants were explored in a semi-structured interview, recorded after the practical aspect.

Acceptability was also explored via a semi-structured interview. Interview questions were developed based on the aims of the semi-structured interviews, which included: to explore user experience and views on using the equipment; whether they found it useful and enjoyable; to identify any concerns; to explore appropriate usage time and setting; and, to gain understanding into barriers and facilitators to future participation. Semi-structured interviews lasted approximately 10 minutes, depending on the amount of information shared by the participant. 
Initial assessment: On study visit 1, prior to use of the game, demographic information was collected including participant age, gender, falls in the last 12 months, walking aid use and number of medications. Assessments of physical function (Short Physical Performance Battery; Guralnik et al. 1994), balance (Berg Balance Scale; Berg et al. 1995), fear of falling (Falls Efficacy ScaleInternational; Yardley et al. 2005) and mood (Geriatric Depression Scale-15 item; Brown et al. 2005) were also collected.

\subsection{Results}

Nine participants (5 female/ 4 male) were involved in user testing of prototype 1; their mean (SD) age was 82.2 (6.3) years. Many participants used a walking aid at home $(n=5)$ or when outside their home $(n=7)$. Six out of nine reported having fallen at least once in the last 12 months; none of these resulted in injury. Four participants had a high risk of falling (Berg Balance Scale score <40), only one participant was considered to have high physical functioning (Short Physical Performance Battery score $>10$ ), and all participants had high fear of falling (Falls Efficacy Scale-International $>28$ ). All participants $(n=9)$ completed a single use of the VR game displayed on screen, while $n=4$ completed an additional single use of the VR game displayed using a VR headset. Results are summarised in Table 1.

Safety and usability: There were no adverse events during use of the system with either viewing medium; however, close supervision was required to ensure safety for both study conditions, particularly during the VR headset study condition. Participants often required the use of hand support and additional instruction during use of the system, this was slightly higher with the VR headset display study condition.

Usability and acceptability: Attitudes to the system were generally positive, and participants' SUS scores indicated acceptable usability of the screen display, but marginally low usability of the VR headset display (Table 1; Figure 3).

Qualitative findings: Preference of the screen version was also evident in the semi-structured interviews, conducted with participants following use of the system. Overall results from the semistructured interviews and comments recorded during use of the system suggested that the participants viewed the VR game, particularly when displayed on screen, as an acceptable mode of exercise; they found the game enjoyable and useful. However, they reported feeling disorientated with the VR headset. Nonetheless, they reported willingness to use the system in the future, confidence in their ability to do so and a preference for use within the day care setting rather than at home. Participants' attitudes to the ACG system were influenced by several factors which were categorised, using an inductive content analysis approach, into three over-arching themes: User experience; motivation; and, ability to participate (Table 2).

\section{DISCUSSION AND CONCLUSIONS}

In line with available guidelines recommending user involvement early in the design process (Fisk et al. 2009; McLaughlin et al. 2012; Uzor et al. 2012; Proffitt and Lange 2013), this study involved older adults throughout the development process to improve users' perceptions of the usability and acceptability of the ACG system. Findings from this study suggested that ACG was safe way to deliver strength and balance exercise to older people. No adverse events were reported during use of the system. Participants unanimously preferred viewing the system displayed on flat screen rather than using a VR headset. While participants reported good levels of usability according to the SUS, they also required high levels of additional support to use the system.

One driver behind this project was to explore the possibility of developing a system that could be used safely and autonomously to meet the needs of more frail older adults who perhaps were unable to attend falls prevention therapy outside of their home. Older adults were involved throughout the development process in order to develop the system to meet their needs and preferences. Although not limited to older adults with high risk of falling, the user testing phase included participants with a history of falls. It documented the level of instruction and supervision needed to use the system to establish whether it could be used at home. Additionally, the use of both quantitative and qualitative measures to explore usability and acceptability of the system explained findings; for example, responses to semi-structured interviews explained the lower SUS score for the VR headset study condition. 
Research to date reports limited information on the usability and acceptability of fully-immersive virtual experiences, such as VR headsets, system in this population. Participants who completed the VR headset study condition reported feeling disorientated, and there was a strong preference of the screen study condition. Ageing is associated with reduced sensitivity of sensory receptors (Goble et al. 2009); this is associated with an increased reliance on visual feedback for postural control (Simoneau et al. 1999; Woollacott and Shumway-Cook 2002). Older adults' experience of VR may be influenced by the disruption or absence of visual feedback from the real environment when wearing the VR headset. Similar findings were also observed in balance-impaired adults aged 59-69 years who reported feeling insecure when playing a VR skiing game displayed using the Oculus Rift VR headset compared with on screen (Epure et al. 2016). Augmented reality may be a way to address this, by enabling older adults to interact with a virtual environment without losing visual feedback of their surroundings.

There were a number of limitations related to the study population and study design. The sample size of this study was small, potentially limiting the ability to draw conclusions based on its findings. However, research on the number of participants required for usability testing indicates the 5-10 participants are sufficient (Faulkner et al. 2003); with some suggestion that multiple small tests are more valuable in allowing iterative changes to be made based on findings with smaller numbers of users (Nielsen et al. 2000). As such, the current sample size is considered to have provided sufficient information to inform the development of the next stage of the study. The findings of this study suggested that older adults perceived ACG to be an acceptable way to deliver exercises for falls prevention; however, recruitment and conduct of the study was in day centres for older people. The specific population and setting may limit the generalisability of the findings to all older adults; however, the study population included those at high risk of falls, thus providing information about the perceptions of those most in need of an ACG intervention for strength and balance exercise.

Although higher for the flat screen version, SUS scores remained at the lower end of acceptable (Figure 3). Implementing user feedback may improve usability of the system. Findings from user testing were used to modify the system. This included choice of display to meet users' preferences; prototype 2 of the system was developed for display only on a flat screen. Findings from user testing of prototype 1, also suggested that a period of familiarisation was of benefit during the introduction of novel technology to older adults. Additionally, it was observed that with single use of the ACG system, high levels of additional instruction were required for use of the system. Therefore, it could be considered that future user testing should evaluate the safety, usability and acceptability of the ACG system with repeated use in older adults.

\section{REFERENCES}

Bangor A, Kortum PT, Miller JT. (2008) An empirical evaluation of the System Usability Scale. International Journal of Human Computer Interactions, 24, pp. 574-594.

Berg, K., Wood-Dauphinee, S. and Williams, J.I., (1995). The Balance Scale: reliability assessment with elderly residents and patients with an acute stroke. Scandinavian Journal of Rehabilitation Medicine, 27(1), 27-36

Bleakley, C.M., Charles, D., Porter-Armstrong, A., McNeill, M.D., McDonough, S.M. and McCormack, B. (2015) Gaming for health: A systematic review of the physical and cognitive effects of interactive computer games in older adults. Journal of Applied Gerontology, 34(3), 166-189.

Brooke, J. (1996) System usability scale (SUS). Usability Evaluation in Industry. London: Taylor and Francis.

Brown, L.M., \& Schinka, J.A. (2005) Development and initial validation of a 15-item informant version of the Geriatric Depression Scale. International Journal of Geriatric Psychiatry, 20(10), 911-918

Brox, E., Konstantinidis, S.T. and Evertsen, G. (2017) User-Centered Design of Serious Games for Older Adults Following 3 Years of Experience with Exergames for Seniors: A Study Design. JMIR serious games, $\mathbf{5}(1)$.

Campbell, A.J. and Robertson, M.C., 2007. Rethinking individual and community fall prevention strategies: a meta-regression comparing single and multifactorial interventions. Age and ageing, 36(6), pp.656-662. 
Epure, P., Gheorghe, C., Nissen, T., Toader, L.O., Macovei, A.N., Nielsen, S.S. and Christensen, D.J.R. (2016) Effect of the Oculus Rift head mounted display on postural stability. International Journal of Child Health and Human Development, 9(3), 343.

Faulkner, L. (2003) Beyond the five-user assumption: Benefits of increased sample sizes in usability testing. Behavior Research Methods, 35(3), 379-383.

Finucane, C., Feeney, J., Nolan, H., O'regan, C., Cronin, H., Kenny, R.A. (2014) The Changing Physical Health of the Over-50s (2009-2012): Findings from The Irish Longitudinal Study on Ageing. Irish Journal of Medical Science, 183, S376-S377.

Fisk, A.D., Rogers, W.A., Charness, N., Czaja, S.J., Sharit, J. (2009) Designing for Older Adults: Principles and Creative Human Factors Approaches, Second Edition, Florida: Human Factors and Aging Series.

Folstein, M.F., Folstein, S.E., \& Mchugh, P.R. (1975) Mini Mental state. A practical method for grading the cognitive state of patients for the clinician. Journal of Psychiatric Research, 12, 189-198

García-Betances, R.I., Waldmeyer, M.T.A., Fico, G. and Cabrera-Umpiérrez, M.F. (2015) A succinct overview of virtual reality technology use in Alzheimer's disease. Frontiers in Aging Neuroscience, 7

Gardner, M.M., Buchner, D.M., Robertson, M.C. and Campbell, A.J. (2001) Practical implementation of an exercise-based falls prevention programme. Age and Ageing, 30(1), 77-83.

Goble, D.J., Coxon, J.P., Wenderoth, N., Van Impe, A. and Swinnen, S.P. (2009) Proprioceptive sensibility in the elderly: degeneration, functional consequences and plastic-adaptive processes. Neuroscience \& Biobehavioral Reviews, 33(3), 271-278.

Guralnik, J.M., Simonsick, E.M., Ferrucci, L., Glynn, R.J., Berkman, L.F., Blazer, D.G., Scherr, P.A., Wallace, R.B. (1994) A short physical performance battery assessing lower extremity function: association with self-reported disability and prediction of mortality and nursing home admission. $J$ Gerontol Med Sci, 49(2), 85-94.

Holmes, D., Charles, D.K., Morrow, P.J., McClean, S. and McDonough, S.M. (2016) Usability and Performance of Leap Motion and Oculus Rift for Upper Arm Virtual Reality Stroke Rehabilitation. In Proceedings of the 11th International Conference on Disability, Virtual Reality \& Associated Technologies. Central Archive at the University of Reading.

Howes, S.C., Charles, D.K., Marley, J., Pedlow, K. and McDonough, S.M., 2017. Gaming for Health: Systematic Review and Meta-analysis of the Physical and Cognitive Effects of Active Computer Gaming in Older Adults. Physical Therapy, 97(12), pp.1122-1137.

Kim, A., Darakjian, N., Finley, J.M. (2017) Walking in fully immersive virtual environments: an evaluation of potential adverse effects in older adults and individuals with Parkinson's disease. Journal of NeuroEngineering and Rehabilitation, 14:1, 16-28

Lanyi, C.S., Brown, D.J., Standen, P., Lewis, J. and Butkute, V., 2012. Results of user interface evaluation of serious games for students with intellectual disability. Acta Polytechnica Hungarica, 9(1), pp.225-245.

Lu, D. Mattiasson, J. (2013) How does Head Mounted Displays affect users' expressed sense of ingame enjoyment, Uppsala universitet Inst. för informationsvetenskap thesis.

Masud T, Morris RO. (2001) Epidemiology of falls. Age Ageing; 30(3), 7.

McLaughlin, A., Gandy, M., Allaire, J. and Whitlock, L. (2012) Putting fun into video games for older adults. Ergonomics in Design, 20(2), 13-22.

National Institute for Health and Care Excellence. (2013). Falls: assessment and prevention of falls in older people CG161. London: NICE

Nielsen, J. and Norman, D.A., 2000. Usability on the Web Isn't a Luxury. Informationweek, (773), 6569.

Newbutt, N., Sung, C., Kuo, H.J. and Leahy, M.J. (2017) The acceptance, challenges, and future applications of wearable technology and virtual reality to support people with autism spectrum disorders. In Recent Advances in Technologies for Inclusive Well-Being, Springer International Publishing, 221-241.

Nilsson, N.C., Serafin, S. and Nordahl, R. (2016) Walking in place through virtual worlds. In International Conference on Human-Computer Interaction, Springer International Publishing, 37-48.

Proffitt, R. and Lange, B., 2013. User centered design and development of a game for exercise in older adults. International Journal of Technology, Knowledge \& Society, 8(5). 
Province, M.A., Hadley, E.C., Hornbrook, M.C., Lipsitz, L.A., Miller, J.P., Mulrow, C.D., Ory, M.G., \& Wolfson, L.I. (1995) The effects of exercise on falls in elderly patients: a preplanned meta-analysis of the FICSIT trials. JAMA, 273(17), 1341-1347.

Riva, G., Wiederhold, B.K. and Gaggioli, A. (2016) Being different. The transformative potential of virtual reality. Annu Rev Cybertherapy Telemed, 14, 1-4

Sherrington, C., Tiedemann, A., Fairhall, N., Close, J. C., \& Lord, S. R. (2011) Exercise to prevent falls in older adults: an updated meta-analysis and best practice recommendations. New South Wales Public Health Bulletin, 22(4), 78-83.

Simoneau, M., Teasdale, N., Bourdin, C., Bard, C., Fleury, M. (1999) Aging and postural control: postural perturbations caused by changing the visual anchor. J Am Geriatr Soc, 47, 235-240.

Standen, P.J., Brown, D.J., Battersby, S., Walker, M., Connell, L., Richardson, A., Platts, F., Threapleton, K. and Burton, A., 2011. A study to evaluate a low cost virtual reality system for home based rehabilitation of the upper limb following stroke. International Journal on Disability and Human Development, 10(4), pp.337-341.

Thomas, S., Reading, J., \& Shephard, R. J. (1992) Revision of the physical activity readiness questionnaire (PAR-Q). Canadian Journal of Sport Sciences.

Uzor, S., Baillie, L. and Skelton, D. (2012) Senior designers: empowering seniors to design enjoyable falls rehabilitation tools. In Proceedings of the SIGCHI Conference on Human Factors in Computing Systems, ACM, 1179-1188.

Woollacott, M., Shumway-Cook, A. (2002) Attention and the control of posture and gait: a review of an emerging area of research. Gait Posture, 16:1-14.

Yardley, L., Beyer, N., Hauer, K., Kempen, G., Piot-Ziegler, C., \& Todd, C. (2005) Development and initial validation of the Falls Efficacy Scale-International (FES-I). Age and Ageing, 34(6), 614-619.

Yardley L, Donovan-Hall M, Francis K, Todd C. (2006) Older people's views of advice about falls prevention: a qualitative study. Health Educ Res. 21(4), 508-17.

Yardley L, Donovan-Hall M, Francis K, Todd C. (2007) Attitudes and beliefs that predict older people's intention to undertake strength and balance training. J Gerontol B Psychol Sci Soc Sci. 62(2), 11925.

Yoo, S. and Kay, J. (2016) VRun: running-in-place virtual reality exergame. In Proceedings of the 28th Australian Conference on Computer-Human Interaction, ACM., 562-566). 